\title{
Investigating the Impact of Group Size on Non-Programming Exercises in CS Education Courses
}

\author{
L.D. Miller \\ Computer Science and Engineering \\ University of Nebraska - Lincoln \\ ldee.miller@gmail.com
}

\author{
Leen-Kiat Soh \\ Computer Science and Engineering \\ University of Nebraska - Lincoln \\ lksoh@cse.unl.edu
}

\author{
Markeya S. Peteranetz \\ Computer Science and Engineering \\ University of Nebraska - Lincoln \\ markeya.dubbs@huskers.unl.edu
}

\begin{abstract}
Computer science (CS) courses are taught with increasing emphasis on group work and with non-programming exercises facilitating peer-based learning, computational thinking, and problem solving. However, relatively little work has been done to investigate the interaction of group work and non-programming exercises because collaborative, non-programming work is usually open-ended and requires analysis of unstructured, natural language responses. In this paper, we consider collaborative, non-programming work consisting of online wiki text from 236 groups in nine different CS1 and higher-level courses at a large Midwestern university. Our investigation uses analysis tools with natural language processing (NLP) and statistical analysis components. First, NLP uses IBM Watson Personality Insights to automatically convert students' collaborative wiki text into a Big Five model. This model is useful as a quality metric on group work since Big Five factors such as Openness and Conscientiousness are strongly related to both academic performance and learning. Then, statistical analysis generates regression models on group size and each Big Five trait that make up the factors. Our results show that increasing group size has a significant impact on collaborative, non-programming work in CS1 courses, but not for such work in higher-level courses. Furthermore, increasing group size can have either a positive or negative impact on the Big Five traits. These findings imply the feasibility of using such tools to automatically assess the quality of non-programming group exercises and offer evidence for effective group sizes.
\end{abstract}

\section{CCS CONCEPTS}

- Social and professional topics $\rightarrow$ Computational thinking; • Applied computing $\rightarrow$ Collaborative learning; E-learning;

\section{KEYWORDS}

CS Education, Non-Programming Exercises, Group Work, Natural Language Processing, Statistical Analysis

Permission to make digital or hard copies of all or part of this work for personal or classroom use is granted without fee provided that copies are not made or distributed for profit or commercial advantage and that copies bear this notice and the full citation on the first page. Copyrights for components of this work owned by others than ACM must be honored. Abstracting with credit is permitted. To copy otherwise, or republish, to post on servers or to redistribute to lists, requires prior specific permission and/or a fee. Request permissions from permissions@acm.org.

SIGCSE'19, February 27-March 2, 2019, Minneapolis, MN, USA

(C) 2019 Association for Computing Machinery.

ACM ISBN 978-1-4503-5890-3/19/2 . \$ $\$ 15.00$

https://doi.org/10.1145/3287324.3287400
ACM Reference Format:

L.D. Miller, Leen-Kiat Soh, and Markeya S. Peteranetz. 2019. Investigating the Impact of Group Size on Non-Programming Exercises in CS Education Courses: Full Paper. In SIGCSE '19: 50th ACM Technical Symposium on Computer Science Education, February 27-March 2, 2019, Minneapolis, MN, USA. ACM, New York, NY, USA, 7 pages. https://doi.org/10.1145/3287324.3287400

\section{INTRODUCTION}

Computer science (CS) courses are taught with increasing emphasis on group work. Group work helps prepare students for an industry where software is developed in teams [4,21,27] such as the popular Agile software development approach [18, 39]. Furthermore, group work helps students develop the "soft skills" necessary for the workplace such as problem solving, self-motivation, leadership, communication, and interpersonal skills. Employers understand the need for these "soft skills" and often value them as much or more than technical skills for employees [18, 27]. Finally, group work has academic benefits for students and instructors in CS with potentially higher retention rates [8] and grades [16] particularly for underrepresented students [12].

At the same time, CS courses are taught with increasing emphasis on moving away from traditional programming exercises. The idea is to provide instruction on the same computational thinking skills, but simplify or remove entirely components which can be frustrating or intimidating to students (especially first year students or those without programming background) such as code syntax [9]. These approaches range from Alice $[9,11]$ and Scratch [5] which use drag-and-drop programming in custom-made integrated development environments (IDEs) to full on CS Unplugged [2] which uses non-programming exercises where students discuss and write exercise responses entirely using natural language $[20,26,36]$. Both these approaches have been shown to provide significant improvements to student grades [11,36].

Despite these separate benefits, relatively little work has been done on the interaction of group work and non-programming exercises. Programming IDEs, including Alice and Scratch, track actions important for group work such as when members uploaded revisions and also analyze whether those revisions ran correctly. On the other hand, actions in non-programming exercises are (1) harder to track since students can communicate through diverse means (e.g., social media) and (2) harder to analyze since exercise content is written in natural language. Without such research, finding an effective group size for non-programming exercises can be operationally difficult. Instead, instructors use ad hoc group sizes for these exercises which dampen the benefits of group work when there is too much work for the group or members can complete the 
exercises solo, or simply just use the same group size as that used in programming exercises. Ultimately, this can increase student dissatisfaction with group work and non-programming exercises even when those exercises are otherwise effective for instruction on computational thinking [24].

Therefore, we propose the use of alternative analysis tools to help researchers and instructors evaluate group work, particularly for non-programming exercises. These tools include two components: (1) advanced natural language processing (NLP) software and (2) statistical analysis dashboard. The NLP software provides quality metrics for the group work written by students, while the dashboard provides statistical analysis on the metrics such as an individual metric broken down by group size. Together, quality metrics and statistical analysis informs researchers on the interaction of group work and non-programming exercises and informs instructors on how group size impacts student activities and performance with those exercises. These tools could also be used to augment other approaches than non-programming exercises such as using NLP to evaluate group discussions in Alice, and to complement group work analysis on programming exercises such as using tracked keystrokes or versions of code check-in, to ultimately form a more comprehensive and meaningful assessment of student group work and learning.

In this paper, we evaluate an analytical process of using NLP and statistical tools on data collected from nine different CS courses at a large Midwestern university, including CS1 and higher-level CS courses. The data collected were from the collaborative text (i.e., online wikis) written by students working in groups to complete non-programming exercises known as the Computational Creativity Exercises [36]. These exercises use active learning, group work, and problem-based learning to teach students creative and computational thinking skills. Students experienced improved individual learning [37] consistent with that expected from collaborative writing [7]. During our analytical process, the NLP component analyzes the collaboratively written texts using IBM Watson Personality Insights [14]. This state-of-the-art NLP software models the collaboratively written texts using a full Big Five personality profile. This profile is useful as a quality metric on group work since Big Five variables such as Openness and Conscientiousness are strongly related to both academic performance and learning [10, 17, 25]. The statistical analysis dashboard provides separate analyses for both the CS1 and higher-level CS courses using R [33]. Both show how all the different Big Five factors are affected by changes in group size.

For this paper, stemming from our overall goal of investigating the impact of group size for non-programming exercises in CS courses, we focus on the following research questions:

- RQ \#1: Does increasing group size have a significant impact on the quality of non-programming exercises-e.g., collaborative wiki essays-for CS1, measured using the Big Five factors?

- RQ \#2: Does group size impact students' work quality differently in higher-level CS courses than in CS1?

The rest of this paper is organized as follows. Section 2 provides background on the non-programming exercises and group work in CS. Section 3 discusses the analytical tools in detail. Section 4 provides the results for the tools evaluated across nine different CS courses with nearly 250 groups. Finally, we conclude and discuss future work.

\section{BACKGROUND AND RELATED WORK}

\subsection{Non-Traditional Exercises}

As previously discussed, CS courses are moving away from using only traditional programming exercises in classrooms. Traditional programming exercises often contain syntax which is frustrating for new students [9] distracting them from developing computational thinking skills which require more than being able to program [40].

Non-traditional exercises range from enhanced programming environment approaches that support syntax-free, drag-and-drop programming such as Alice $[9,11]$ and Scratch $[5]$ to full on CS Unplugged approaches that do away with programming completely. These latter approaches replace programming with natural language exercises [20, 26, 36]. Both enhanced programming environments and CS Unplugged approaches are effective and have been shown to improve student grades [11, 36].

Meanwhile, the original focus of CS Unplugged was nonprogramming exercises for K-12 students where instructors lacked the time, computers, or experience to use traditional programming exercises [2, 3, 26]. Nevertheless, interest in CS Unplugged has steadily increased at the college-level for several reasons: (a) nonprogramming exercises are easier to understand for non-majors and students with diverse backgrounds [6], (b) non-programming exercises are easier to deploy and grade in large sized courses and massive open online courses [20], and (c) non-programming exercises are effective at providing instruction on computational thinking skills important for college-level courses [36].

This paper focuses on non-programming exercises as grading these exercises in large classrooms is challenging and assessment based on this type of exercises has been less investigated. Having said that, our proposed analytical process could also be used for, say, enhanced programming environment exercises. As an example, the analytical tools could analyze group discussions in Alice to form a more meaningful assessment of student group work and learning. We will return to this discussion in Section 5 as future work.

\subsection{Computational Creativity Exercises}

For this paper, we used the data collected from students completing non-programming exercises called the computational creativity exercises (CCEs) [23]. In these exercises, group members used an online wiki which functions similarly to any collaborative writing assignment: each member could add to the wiki or edit work written by other group members. Collaborative writing has long been known to improve (individual) student learning [7]. Recent work has also reported the benefits of using collaborative writing in CS courses [1,32].

CCEs expand on collaborative writing with exercises designed to boost both creative and computational thinking skills using a combination of problem solving, written analysis, and reflection. Students working in groups on these CCEs experienced improved individual learning in their CS courses [37]. Each CCE contains four standard components: (1) Objectives describing the computational thinking 
skills covered in that exercise (such as abstraction); (2) Tasks describing the individual tasks that each student in the group must complete; (3) Light Bulbs providing direct connections between exercise tasks and CS topics; and (4) Questions asking open-ended analysis and reflection questions for group members to discuss.

We evaluated six CCEs previously deployed to both CS1 and higher-level CS courses. The descriptions for those exercises are taken from [37]: (1) Cipher: Devise an encoding scheme to encode questions for other teams to compete to decode; (2) Everyday Object: Describe inputs, outputs, and functionalities for an existing object (e.g., nail clippers); (3) Exploring: Explore an existing location and document the senor stimuli observed there; (4) Pathfinding: Create a step-by-step set of instructions to create a quilt pattern; (5) Storytelling: Write story chapters independently and then work as a team to resolve conflicts and inconsistencies; and (6) Thinking Inside the Box: Test an alien machine without causing harm while attempting to reveal the functionalities of the machine.

\subsection{Group Work}

Group work has been studied for quite some time both (a) in K-12 and college level courses and (b) for CS and other fields. Results show that group work improves student achievement for K-12 and college [19]. Compared to working individually, group work in CS courses allow students to achieve higher retention rates [8] and grades [16] particularly for underrepresented students [12] Furthermore, for CS, group work is important because modern soft-ware is developed in teams [4,21,27] such as the popular Agile soft-ware development approach [18, 39]. As previously mentioned, team development requires individuals with "soft skills" such as problem solving, self-motivation, leadership, communication, and interpersonal skills. Employers value these "soft skills" as much or more than technical skills for employees [18, 27].

Researchers have studied many different approaches for adding group work to CS courses [12]. For traditional programming exercises, pair programming has been studied extensively with groups of 2 students: one student acting as the driver controlling the keyboard and mouse while the other acts as the navigator [38]. Group work has also been previously tried for non-traditional exercises, but instructors tend to use ad hoc group sizes (such as simply allowing students to pick the groups) and have not extensively analyzed exercise content produced by these groups. Examples include group work designed for peer support with group sizes of 2-3 [15] and group work on software development projects with group sizes of 4-6 students [21] and 5 students [34].

Much less work has been done on investigating the impact of group sizes. Larger groups are recommended on semester-long projects involving students from multiple classes [27]. However, results on whether larger groups are better than smaller groups are often contradictory. For example, [30] found that students preferred working in discussion groups of size 2 over size 4 whereas [13] reported a significant positive relationship between (increasing) group size and higher level knowledge construction in groups.

\section{ANALYSIS TOOLS}

This section describes the (1) data collection, (2) advanced natural language processing (NLP), and (3) statistical analysis for our proposed analysis tools. As previously mentioned, the IBM Watson Personality Insights uses NLP to derive from collaboratively written text the set of Big Five personality profiles. Although Big Five profiles are designed to be intuitive (e.g., higher imagination is usually preferred), connecting the results to previous research on group work requires a working understanding of the Big Five. Therefore, we provide a detailed description of the Big Five traits used after describing Personality Insights.

\subsection{Data Collection}

The data collection is based on the deployment of the CCEs previously described (Section 2.2) to nine different CS courses at a large Midwestern university.

The nine different CS courses include six CS1 courses and three higher-level CS courses. The six CS1 courses include all the different versions available at that university including CS1 designed for (a) CS majors, (b) engineering majors, and (c) honors students in a $\mathrm{CS} /$ business crossover program. The three higher-level CS courses include intermediate (year 2-3) courses covering CS hardware and algorithm design.

Students were required to complete the CCEs with collaborative writing essays using an online wiki. The CCEs were graded and counted collectively as $5 \%$ of students' course grade. Students were allowed to form their own groups for the CCEs using the online wiki, but random assignment and group merging services were also available. In particular, group merging was used to keep the groups from dropping in size as students dropped from a course during the semester.

In total, we consider collaborative writing essays on the CCEs collected from 149 different groups in CS1 and 87 different groups in higher-level CS courses. The analysis tools preprocess the essay content automatically to remove HTML and other special characters. And the essays are stored with only group names as identifiers to protect the privacy of students.

\subsection{Advanced Natural Language Processing}

Here we provide a description on the IBM Watson Personality Insights and the Big Five traits used.

Personality Insights. IBM Watson Personality Insights is a natural language processing module of the well-known Watson AI system which defeated human experts on the Jeopardy! game show in 2011. Personality Insights uses state-of-the-art natural language processing (NLP) to derive from text the set of Big Five personality profiles [14]. First, Personality Insights uses a word-embedded technique called GloVe [28] to obtain a vector representation for the text. Then, this vector is fed into a machine learning algorithm trained on thousands of users which predicts Big Five traits for the text. Although details on the machine learning algorithm are proprietary, its validity was established by comparing the Big Five profiles predicted by the algorithm to Big Five profiles based on surveys [31,35]. More specifically, the mean absolute error and correlation validate that Big Five profiles from Personality Insights are quite similar to those for survey-based Big Five profiles on the same users.

Personality Insights operates as part of the IBM cloud and provides a relatively simple abstract programming interface (API) for 
use. The machine learning algorithm is already trained and immutable. As such, the order and content of the text uploaded have no impact on the Big Five model predicted. In the results below, we uploaded each essay one at a time to the cloud using the API. After running the algorithm, Personality Insights returned a separate Big Five profile for each essay with different traits.

Big Five. The five-factor personality model is most commonly assessed using a complex questionnaire such as NEO Personality Inventory-Revised [22]. This questionnaire has a five-point Likert scale paired with 240 items that assess 30 different traits (sometimes called facets). Each set of six traits define one of the five factors important for this personality and behavior model: Openness, Conscientiousness, Extraversion, Agreeableness, and Emotional Range (also called Neuroticism).

For this initial work, we evaluate our analysis tools on three of the five main factors: Openness, Conscientiousness, and Agreeableness. As personality traits reflected in individuals, these three factors have been consistently connected to both academic performance and learning [10, 17, 25]. As such, these factors are probably the most immediately interesting for instructors and researchers. (Evaluation on the other two, more emotional, factors is discussed in the future work.) The 18 different traits corresponding to the three factors are described in detail in Table 1 with descriptions paraphrased from [14] and [22].

Finally, as previously discussed, IBM Watson Personality Insights uses a machine learning approach to predict the scores for the Big Five model [14]. These scores are given on a percentile range of 0 100 comparing the traits to those found in the thousands of writing samples used for training. As such, an Imagination score of $90 \%$ in the results below means that the essay content is more imaginative than $90 \%$ of the baseline (rather than $90 \%$ imaginative which would be nonsensical).

\subsection{Statistical Analysis}

The analysis tools automatically calculate statistics on the Big Five traits using the $\mathrm{R}$ programming language. $\mathrm{R}$ is a free and opensource language which includes a large range of statistical models and allows for data organization, statistical analysis, and graphical representation using the same software. The results below use separate regression models to measure the impact of changing group size on each Big Five trait. The model provides a slope for each trait along with statistical significance (with F-test).

\section{RESULTS}

Tables 2-3 shows the results of our analysis across nine different CS courses with 236 groups. Both tables provide results measuring the impact of group size on Big Five traits for the Openness, Conscientiousness, and Agreeableness factors. Table 2 provides those results for CS1, based on 149 groups, while Table 3 them for higher-level CS courses, based on 87 groups. Both tables are organized in the same way. First, the average, predicted score for each Big Five trait is given separately for groups sizes 1-5 (S1-S5). The largest value for a single trait has a blue background, while the smallest has a red background. Larger groups (size 6-7) are not included because there are fewer than five such groups and they are only found in
Table 1: Descriptions of traits for Openness, Conscientiousness, and Agreeableness-Big Five factors used in the results.

\begin{tabular}{|l|l|}
\hline Openness & Individuals who score high... \\
\hline Adventurousness & $\begin{array}{l}\text { Are eager to try new activities and experi- } \\
\text { ence different things. }\end{array}$ \\
\hline Artistic interests & Love beauty, both in art and in nature. \\
\hline Emotionality & Have good awareness of their own feelings. \\
\hline Imagination & View the real world as often too ordinary. \\
\hline Intellect & $\begin{array}{l}\text { Are intellectually curious and tend to think in } \\
\text { symbols and abstractions. }\end{array}$ \\
\hline Authority challenging & $\begin{array}{l}\text { Have a readiness to challenge authority, con- } \\
\text { vention, and traditional values. }\end{array}$ \\
\hline Conscientiousness & Individuals who score high... \\
\hline Achievement striving & Try hard to achieve excellence. \\
\hline Cautiousness & $\begin{array}{l}\text { Are disposed to think through possibilities } \\
\text { carefully before acting. }\end{array}$ \\
\hline Dutifulness & Have a strong sense of duty and obligation. \\
\hline Orderliness & Are well-organized, tidy, and neat. \\
\hline Self discipline & $\begin{array}{l}\text { Have the self-discipline to persist at difficult } \\
\text { or unpleasant tasks until they are completed. }\end{array}$ \\
\hline Self efficacy & $\begin{array}{l}\text { Confident in their ability to accomplish } \\
\text { things. }\end{array}$ \\
\hline Agreeableness & Individuals who score high... \\
\hline Altruism & $\begin{array}{l}\text { Find that helping others is genuinely reward- } \\
\text { ing. }\end{array}$ \\
\hline Cooperation & Dislike confrontation. Willing to compromise. \\
\hline Modesty & Are unassuming, self-effacing, and humble. \\
\hline Uncompromising & $\begin{array}{l}\text { See no need for pretense or manipulation } \\
\text { when dealing with others. }\end{array}$ \\
\hline Sympathy & Are tender-hearted and compassionate. \\
\hline Trust & $\begin{array}{l}\text { Assume that most people are fundamentally } \\
\text { fair, honest, and have good intentions. }\end{array}$ \\
\hline
\end{tabular}

one course. Second, the slope for the regression model with standardized coefficients and statistical significance using a F-test (Reg. and Sig.) are given for each Big Five trait. This model is run using all scores for groups sizes 1-5 (not just the average scores).

\subsection{CS1 Results}

Table 2 provides considerably interesting analysis tool results for the $\mathrm{CS} 1$ courses. First, overall, group size seems to have a significant impact on all three Big Five factors (Openness, Conscientiousness, and Agreeableness) with statistical significance on $4 / 6$ of the traits for each factor. As previously mentioned, these factors are important for CS education because they have been consistently connected to academic performance and learning $[10,17,25]$. That being said, increasing group size impacts the Big Five factors in decidedly different ways.

Openness. At first, increasing group size seems to have a mixed impact on Openness traits. Imagination and Emotionality traits both go up significantly with increasing group size, while Adventurousness and Authority challenging both go down significantly. One possible interpretation is larger groups were more likely to revise and "soften" the bold aspects of language that are scored as Adventurous or Authority challenging. Larger groups result in written essays which are more empathetic and imaginative, but 
also remain more focused on the topic under discussion for the assignment. These results are particularly interesting since students work together on the CCEs remotely using an online wiki rather than directly in class. Larger groups could act a kind of remote peer pressure on students encouraging them to take the CCEs seriously and make insightful revisions to avoid upsetting a larger number of their peers. At this point, we conclude that increasing group size improves most Openness traits on the CCE non-programming exercises. Future work investigating the impact of group size on Openness for groups working together remotely vs. directly in the classroom should be considered.

Conscientiousness. Increasing group size reduces most Conscientiousness traits on the CCE non-programming exercises. Achievement striving, Orderliness, Self discipline, and Self efficacy all go down significantly with increasing group size. To an extent, these results should be expected since the written essays from larger groups are likely going to be less cohesive, less smooth, and less logically consistent than essays written by a single individual. The CCE non-programming exercises place emphasis on getting all students to contribute to the written essays. However, in larger groups, members might end up placing less emphasis making sure those essays are rigorously proofed and edited. Larger groups and lack of editing combine to lower Conscientiousness scores from Personality Insights which compares against the writing samples used for training. In a way, to improve Conscientiousness, this type of non-programming exercises should place emphasis on making sure or incentivizing that content is proofed and edited. Future work investigating the impact of group size on Conscientiousness should consider including a group leader who guides the essay writing including editing.

Agreeableness. Increasing group size has a mixed impact on Agreeableness traits for the CCEs. Altruism and Sympathy both go up significantly with increasing group size, while Cooperation and Uncompromising go down significantly. Altruism and Sympathy and Uncompromising are arguably more important for CCEs to help students describe, document, and create in the written essays because there are more viewpoints involved in the writing process when the group size is larger. We speculate that the reduction in Cooperation make sense as larger groups will have more voices which are less cohesive leading to a final product that appears to be more confrontational. As previously mentioned, the CCEs emphasize individual contribution and do not explicitly encourage electing a group leader guiding the essay writing. Meanwhile, we realized that the online wiki also has a separate discussion forum from the written essay. When we went back to look at the forum, we found that most students actually used social media for discussion (e.g., Facebook). Would including those discussions improve the scores on Cooperation and Uncompromising as group size increases? Future work investigating the impact of group size on Agreeableness' traits should include social media discussions.

Discussion. The results in Section 4.1 show that increasing group sizes have a significant impact on non-programming exercises for CS1 measured using the Big Five factors (RQ \#1). Increasing group sizes (1) improves most Openness traits, (2) reduces most
Table 2: CS1 regression results measuring the impact of group size on Big Five traits. S1-S5 gives trait for group sizes with $\max$ (blue) and min (red), Reg. gives the slope for model, Sig. gives statistical significance $\left({ }^{*} \leq 0.1,{ }^{* *} \leq 0.05,{ }^{* *} \leq 0.01\right)$

\begin{tabular}{|l|c|c|c|c|c|c|c|}
\hline Openness & S1 & S2 & S3 & S4 & S5 & Reg. & Sig. \\
\hline Adventurousness & 0.42 & 0.36 & 0.31 & 0.27 & 0.22 & -0.05 & $* * *$ \\
\hline Artistic interests & 0.55 & 0.60 & 0.56 & 0.64 & 0.65 & 0.02 & \\
\hline Emotionality & 0.26 & 0.38 & 0.37 & 0.54 & 0.54 & 0.07 & $* * *$ \\
\hline Imagination & 0.67 & 0.73 & 0.78 & 0.84 & 0.85 & 0.05 & $* * *$ \\
\hline Intellect & 0.97 & 0.98 & 0.97 & 0.98 & 0.98 & 0.00 & \\
\hline Authority challenging & 0.88 & 0.88 & 0.83 & 0.77 & 0.77 & -0.03 & $* * *$ \\
\hline Conscientiousness & S1 & S2 & S3 & S4 & S5 & Reg. & Sig. \\
\hline Achievement striving & 0.43 & 0.42 & 0.36 & 0.25 & 0.24 & -0.05 & $* * *$ \\
\hline Cautiousness & 0.76 & 0.80 & 0.73 & 0.76 & 0.76 & 0.00 & \\
\hline Dutifulness & 0.44 & 0.42 & 0.42 & 0.48 & 0.44 & 0.00 & \\
\hline Orderliness & 0.51 & 0.44 & 0.42 & 0.36 & 0.37 & -0.03 & $* * *$ \\
\hline Self discipline & 0.32 & 0.24 & 0.22 & 0.09 & 0.10 & -0.06 & $* * *$ \\
\hline Self efficacy & 0.51 & 0.45 & 0.38 & 0.25 & 0.27 & -0.06 & $* * *$ \\
\hline Agreeableness & S1 & S2 & S3 & S4 & S5 & Reg. & Sig. \\
\hline Altruism & 0.41 & 0.48 & 0.47 & 0.58 & 0.56 & 0.04 & $* * *$ \\
\hline Cooperation & 0.55 & 0.55 & 0.49 & 0.50 & 0.44 & -0.03 & $* *$ \\
\hline Modesty & 0.51 & 0.54 & 0.57 & 0.56 & 0.54 & 0.01 & \\
\hline Uncompromising & 0.51 & 0.52 & 0.43 & 0.40 & 0.41 & -0.03 & $* *$ \\
\hline Sympathy & 0.57 & 0.69 & 0.65 & 0.83 & 0.80 & 0.06 & $* * *$ \\
\hline Trust & 0.43 & 0.42 & 0.41 & 0.37 & 0.38 & -0.01 & \\
\hline
\end{tabular}

Conscientiousness traits, and (3) has a mixed impact on Agreeableness traits. These results have different takeaways for instructors and researchers.

First, instructors can use these results as a guide for tradeoffs in group size on non-programming exercises. The instructor can decide which traits are most important on the exercises and set the group size accordingly. As an example, exercises which emphasize Imagination should use larger groups, while those which emphasize Orderliness should use smaller groups.

Second, researchers should use these results as a starting point to supplement or complement their designs. Although using our analysis tools to evaluate non-programming exercises content using Big Five factors seems promising, some results are influenced by design choices in the CCEs (e.g., no group leader). As such, additional work must be done to further evaluate (a) the impact of group size on the Big Five factors (as previously suggested) and (b) the analysis tools on other non-programming exercises with different design choices.

\subsection{Higher-Level Results}

Table 3 provides analysis tool results for the higher-level courses. Overall, group size does not have a significant impact on all three Big Five factors (Openness, Conscientiousness, and Agreeableness) with statistical significance on only $2 / 18$ traits. We might be tempted to speculate that group work has nothing to do with these results: 
Table 3: Higher-Level CS regression results measuring the impact of group size on Big Five traits. S1-S5 gives trait for group sizes with max (blue) and min (red), Reg. gives the slope for model, Sig. gives statistical significance $\left({ }^{*} \leq 0.1\right.$, * $\leq 0.05,{ }^{* *} \leq \mathbf{0 . 0 1}$ ).

\begin{tabular}{|l|c|c|c|c|c|c|}
\hline Openness & S1 & S2 & S3 & S4 & Reg. & Sig. \\
\hline Adventurousness & 0.51 & 0.45 & 0.44 & 0.53 & 0.00 & \\
\hline Artistic interests & 0.52 & 0.65 & 0.63 & 0.66 & 0.04 & \\
\hline Emotionality & 0.19 & 0.17 & 0.17 & 0.13 & -0.02 & \\
\hline Imagination & 0.51 & 0.74 & 0.72 & 0.67 & 0.05 & \\
\hline Intellect & 0.97 & 0.98 & 0.97 & 0.99 & 0.00 & \\
\hline Authority challenging & 0.89 & 0.94 & 0.95 & 0.92 & 0.01 & \\
\hline Conscientiousness & S1 & S2 & S3 & S4 & Reg. & Sig. \\
\hline Achievement striving & 0.56 & 0.42 & 0.46 & 0.47 & -0.02 & \\
\hline Cautiousness & 0.87 & 0.77 & 0.80 & 0.84 & -0.01 & \\
\hline Dutifulness & 0.57 & 0.35 & 0.37 & 0.40 & -0.05 & $*$ \\
\hline Orderliness & 0.65 & 0.52 & 0.56 & 0.61 & -0.01 & \\
\hline Self discipline & 0.50 & 0.29 & 0.33 & 0.36 & -0.04 & \\
\hline Self efficacy & 0.59 & 0.52 & 0.53 & 0.51 & -0.02 & \\
\hline Agreeableness & S1 & S2 & S3 & S4 & Reg. & Sig. \\
\hline Altruism & 0.46 & 0.34 & 0.33 & 0.28 & -0.06 & $* * *$ \\
\hline Cooperation & 0.73 & 0.52 & 0.55 & 0.59 & -0.04 & \\
\hline Modesty & 0.66 & 0.51 & 0.50 & 0.56 & -0.04 & \\
\hline Uncompromising & 0.66 & 0.44 & 0.47 & 0.53 & -0.04 & \\
\hline Sympathy & 0.56 & 0.57 & 0.49 & 0.50 & -0.03 & \\
\hline Trust & 0.43 & 0.45 & 0.43 & 0.40 & -0.01 & \\
\hline
\end{tabular}

the CCEs are simply too easy for higher-level CS students. However, recent work from others has shown that the CCEs helped with learning in higher-level students [29]. As such, the differences between Tables 2-3 point to a fundamental difference in group work between CS1 and higher-level CS students when using the non-programming exercises. Older students potentially have more experience with group work and are less affected by the size of the group they are working in.

Openness. Students in higher-level courses (1) have very high levels of Authority challenging and (2) Adventurousness and $\mathrm{Au}-$ thority challenging both increase with group size. Imagination does increase at $\mathrm{S} 2$, but then actually goes down as group size increases. As previously mentioned, increasing Adventurousness and Authority challenging can be undesirable for assignments. In general, where larger groups are beneficial for Openness in CS1 students, the opposite may be true for students in higher-level courses. One explanation is that these students, having gone through their first or second year of courses, are more independent and have better problem solving skills and more structured reasoning processes. Thus, working in a smaller or a larger group does not impact the quality of the collaborative wiki essay significantly.

Conscientiousness and Agreeableness. Students in higherlevel courses have the highest values for traits at S1-S2 before an observable decline in all traits across both factors. These results provide additional evidence that increasing group size dose not impact the quality of these students' collaborative work, or if there is impact, it appears to be more negative than positive.

Discussion. The results in Section 4.2 show that group size does not impact higher-level CS courses as much as CS1 (RQ \#2). The difference is probably in how students view group work since students in higher-level courses still benefit from using the exercises [29]. We speculate that students in higher-level courses are more adept at working independently and have stronger knowledge on the subject matter that they are capable of producing similar quality of work by themselves or as a team. Nevertheless, these results demonstrate how the analysis tools could provide important information to instructors allowing for redesigning of group assignments to invoke more meaningful teamwork.

\section{CONCLUSIONS}

CS courses are being taught with increasing emphasis on group work and non-programming exercises, but little work has been done investigating the impact of group size on these exercises. The reason is that student responses to non-programming exercises are often written in natural language making analysis difficult. We propose analysis tools which use natural language processing and statistical analysis to evaluate collaborative, non-programming work effectively. These tools use IBM Watson Personality Insights to convert the natural language into a Big Five profile and then generates statistical models on the group size and Big Five traits on factors important for learning (e.g., Openness). Our results show that increasing group size seems to have a significant impact on non-programming exercises for CS1. Also, increasing group size does not have as much of an impact on higher-level CS courses as it does in CS1. Instead, there appears to be a difference between students working in a group or not in a group.

We have only started evaluating group work on non-programming exercises. We have already mentioned future work on the impact of groups working remotely vs. directly, the presence of a group leader, and the inclusion of social media discussions. Also, while the CCEs provide general benefits for all students [36], we also intend to investigate whether presence in groups with high Big Five scores correlates with individual performance in the CS classroom in the same way that prior research has shown the traits in individuals are associated with learning outcomes.

Finally, the analysis tools using Personality Insights have a huge potential for use with other research areas. First, the tools could be used for the enhanced programming environments such as Alice and Scratch. In addition to monitoring student programming, these environments could obtain a more complete picture by analyzing the quality of group discussions. Second, massive open online courses could use these tools for the analysis of group discussions and exercise content which requires natural language responses.

\section{ACKNOWLEDGMENTS}

This work was partially supported by the National Science Foundation with grants no. 1122956 and 1431874 . Further support was provided with a UNL Phase II Pathways to Interdisciplinary Research Centers grant. 


\section{REFERENCES}

[1] Oluwabunmi Adewoyin, Kewen Wu, and Julita Vassileva. 2015. Exploiting the Use of Wikis to Support Collaborative Writing: A Case Study of an Undergraduate Computer Science Class. In Proceedings of the International Conference on Collaboration and Technology (CRIWG 2015). 111-123.

[2] Tim Bell, Jason Alexander, Isaac Freeman, and Mick Grimley. 2009. Computer Science Unplugged: school students doing real computing without computers. New Zealand Journal of Applied Computing \& Information Technology 13, 1 (2009), $20-29$.

[3] Tim Bell, Paul Curzon, Quintin Cutts, Valentina Dagiene, and Bruria Haberman 2011. Overcoming Obstacles to CS Education by Using Non-programming Outreach Programmes. In Proceedings of International Conference on Informatics in Schools: Situation, Evolution, and Perspectives (ISSEP'11). 71-81.

[4] Jürgen Börstler and Thomas Hilburn. 2016. Team Projects in Computing Education. ACM Transactions on Computing Education 16, 2 (2016), 1-4.

[5] Karen Brennan and Mitchel Resnick. 2012. New frameworks for studying and assessing the development of computational thinking. In Proceedings of American Educational Research Association meeting. Vancouver, British Columbia, Canada, $1-25$.

[6] Richard Brown and Elizabeth Shoop. 2011. Modules in Community: Injecting More Parallelism Into Computer Science Curricula. In Proceedings of the 42rd ACM Technical Symposium on Computer Science Education (SIGCSE'11). Dallas, Texas, USA, 447-452.

[7] Rafael Calvo, Stephen T. O'Rourke, Janet Jones, Kalina Yacef, and Peter Reimann 2011. Collaborative Writing Support Tools on the Cloud. IEEE Transactions on Learning Technology 4, 1 (2011), 81-97.

[8] Adam Carter, Christopher Hundhausen, and Olusola Adesope. 2017. Blending Measures of Programming and Social Behavior into Predictive Models of Student Achievement in Early Computing Courses. ACM Transactions on Computing Education 17, 3 (2017), 1-20.

[9] Stephen Cooper, Wanda Dann, and Randy Pausch. 2003. Teaching Objects-first In Introductory Computer Science. In Proceedings of the 34th ACM Technical Symposium on Computer Science Education (SIGCSE'03). Reno, Nevada, USA, 1-5.

[10] Holger Danielsiek and Jan Vahrenhold. 2016. Stay on These Roads: Potential Factors Indicating Students' Performance in a CS2 Course. In Proceedings of the 47th ACM Technical Symposium on Computer Science Education (SIGCSE'16). Memphis, Tennessee, USA, 12-17.

[11] Wanda Dann, Dennis Cosgrove, Don Slater, Dave Culyba, and Steve Cooper. 2012. Mediated transfer: Alice 3 to Java. In Proceedings of the 43rd ACM Technical Symposium on Computer Science Education (SIGCSE'12). Raleigh, North Carolina, USA, 141-146.

[12] Scott Grissom, Renée McCauley, and Laurie Murphy. 2018. How Student Centered is the Computer Science Classroom? A Survey of College Faculty. ACM Transactions on Computing Education 18, 1 (2018), 1-27.

[13] Khe Foon Hew and Wing Sum Cheung. 2911. Higher-level knowledge construction in asynchronous online discussions: an analysis of group size, duration of online discussion, and student facilitation techniques. Instructional Science 39 (2911), 303-319.

[14] IBM Cloud Docs. 2017. The science behind the service. IBM. https://console. bluemix.net/docs/services/personality-insights/science.html\#science

[15] Ville Isomöttönen and Ville Tirronen. 2017. Flipping and BlendingâĂŤAn Action Research Project on Improving a Functional Programming Course. ACM Transactions on Computing Education 17, 1 (2017), 1-35.

[16] Erkki Kaila, Einari Kurvinen, Erno Lokkila, and Mikko-Jussi Laakso. 2016. Redesigning an Object-Oriented Programming Course. ACM Transactions on Computing Education 16, 4 (2016), 1-21.

[17] Meera Komarraju, Steven Karau, Ronald Schmeck, and Alen Avdic. 2011. The Big Five personality traits, learning styles, and academic achievement. Personality and Individual Differences 51 (2011), 472-477.

[18] David Largent. 2016. Measuring and Understanding Team Development by Capturing Self-assessed Enthusiasm and Skill Levels. ACM Transactions on Computing Education 16, 2 (2016), 1-27.

[19] Yiping Lou, Philip Abrami, John Spence, Catherine Poulsen, Bette Chambers, and Sylvia Apollonia. 1996. Within-Class Grouping: A Meta-Analysis. Review of Educational Research 66, 4 (1996), 423-458.

[20] Mary Lou Maher, Celine Latulipe, Heather Lipford, and Audrey Rorrer. 2015 Flipped Classroom Strategies for CS Education. In Proceedings of the 46th ACM Technical Symposium on Computer Science Education (SIGCSE'15). Kansas City, Missouri, USA, 218-223.

[21] Linda Marshall, Vreda Pieterse, Lisa Thompson, and Dina Venter. 2016. Exploration of Participation in Student Software Engineering Teams. ACM Transactions on Computing Education 16, 2 (2016), 1-38.

[22] Robert McCrae and Paul Costa. 2010. NEO inventories professional manual. Psychological Assessment Resources, Odessa, Florida, USA.

[23] L.D. Miller, Leen-Kiat Soh, Vlad Chiriacescu, Elizabeth Ingraham, Duane Shell, and Melissa Hazley. 2014. Integrating computational and creative thinking to improve learning and performance in CS1. In Proceedings of the 45th ACM Technical
Symposium on Computer Science Education (SIGCSE'18). Atlanta, Georgia, USA, 475-480.

[24] L.D. Miller, Leen-Kiat Soh, Vlad Chiriacescu, Elizabeth Ingraham, Duane Shell, Stephen Ramsey, and Melissa Hazley. 2013. Improving learning of computational thinking using creative competency exercises in college CS-1 computer science courses. In Proceedings of 2013 IEEE Frontiers in Education Conference (FIE'13). 1426-1432.

[25] Erik Noftle and Richard Robins. 2007. Personality Predictors of Academic Outcomes: Big Five Correlates of GPA and SAT Scores. Fournal of Personality and Social Psychology, 93, 1 (2007), 116-130.

[26] Youwen Ouyang, Katherine Hayden, and Julie Remold. 2018. Introducing Computational Thinking through Non-Programming Science Activities. In Proceedings of the 49th ACM Technical Symposium on Computer Science Education (SIGCSE'18). Baltimore, Maryland, USA, 308-313.

[27] Robert Pastel, Marika Seigel, Wei Zhang, and Alex Mayer. 2015. Team Building in Multidisciplinary Client-Sponsored Project Courses. ACM Transactions on Computing Education 15, 4 (2015), 1-24.

[28] Jeffrey Pennington, Richard Socher, and Christopher Manning. 2014. GloVe: Global Vectors for Word Representation. In Proceedings of the 2014 Conference on Empirical Methods in Natural Language Processing (EMNLP). 1532-1543.

[29] Markeya Peteranetz, Shiyuan Wang, Duane Shell, Abraham Flanigan, and LeenKiat Soh. 2018. Examining the Impact of Computational Creativity Exercises on College Computer Science Students' Learning, Achievement, Self-Efficacy, and Creativity. In Proceedings of the 49th ACM Technical Symposium on Computer Science Education (SIGCSE'18). Baltimore, Maryland, USA, 155-160.

[30] Johanna Pirker, Maria Riffnaller-Schiefer, and Christian Gütl. 2014. Motivational Active Learning âĂŞ Engaging UniversityStudents in Computer Science Education. In Proceedings of the 2014 conference on Innovation \& technology in computer science education (ITiCSE'14). Uppsala, Sweden, 297-302.

[31] Barbara Plank and Dirk Hovy. 2015. Personality Traits on Twitter -or- How to Get 1,500 Personality Tests in a Week. In Proceedings of the 6th Workshop on Computational Approaches to Subjectivity, Sentiment and Social Media Analysis (WASSA 2015). 92-98.

[32] Elvira Popescu, Mihai Dascalu, Alex Becheru, Scott Crossley, and Stefan TrausanMatu. 2016. Predicting Student Performance and Differences in Learning Styles based on Textual Complexity Indices applied on Blog and Microblog Posts. In Proceedings of IEEE 16th International Conference on Advanced Learning Technologies (ICALT 2016). 184-188.

[33] R Core Team. 2015. R: A Language and Environment for Statistical Computing. R Foundation for Statistical Computing, Vienna, Austria. https://www.R-project. org/

[34] Kurt Schneider, Olga Liskin, Hilko Paulsen, and Simone Kauffeld. 2015. Media, Mood, and Meetings: Related to Project Success? ACM Transactions on Computing Education 15, 4 (2015), 1-33.

[35] Andrew Schwartz, Johannes Eichstaedt, Margaret Kern, Lukasz Dziurzynski, Stephanie Ramones, Megha Agrawal, Achal Shah, Michal Kosinksi, and David Stillwell. 2013. Personality, Gender, and Age in the Language of Social Media: The Open-Vocabulary Approach. PLoS One 8, 9 (2013), 1-16.

[36] Duane Shell and Leen-Kiat Soh. 2013. Profiles of Motivated Self-Regulation in College Computer Science Courses: Differences in Major Versus Required Nonmajor Courses. Fournal of Science Education and Technology 22 (2013), 899-913.

[37] Duane Shell, Leen-Kiat Soh, Abraham Flanigan, Markeya Peteranetz, and Elizabeth Ingraham. 2017. Improving Students' Learning and Achievement in CS Classrooms through Computational Creativity Exercises that Integrate Computational and Creative Thinking. In Proceedings of the 48th ACM Technical Symposium on Computer Science Education (SIGCSE'17). Seattle, Washington, USA, 543-548.

[38] Karthikeyan Umapathy and Albert Ritzhaupt. 2017. A Meta-Analysis of PairProgramming in Computer Programming Courses: Implications for Educational Practice. ACM Transactions on Computing Education 17, 4 (2017), 1-13.

[39] Rebecca Vivian, Katrina Falkner, Nickolas Falkner, and Hamid Tarmazdi. 2016. A Method to Analyze Computer Science Students' Teamwork in Online Collaborative Learning Environments. ACM Transactions on Computing Education 16, 2 (2016), 1-28

[40] Jeannette Wing. 2006. Computational Thinking. Commun. ACM 49, 3 (2006), 33-35. 